\section{IGH rearrangement in myeloid neoplasms}

Though immunoglobulin genes are typically expressed in B lymphocytes, recent studies found ectopic immunoglobulin expression in non-B-cell tumor cells including acute myeloid leukemia (AML). The immunoglobulin genes, including immunoglobulin heavy chain genes $(I G H)$, light kappa ( $\kappa)$ chain genes (IGK) and light lambda $(\lambda)$ chain genes $(I G L)$, are frequently rearranged in B-cell tumors. These rearrangements result in a juxtaposition of IG enhancers to the vicinity of onco- genes such as MYC and BCL2, leading to their overexpression and activation. Here, we found two IGH rearrangements in myeloid tumors, including an IGH-MECOM in a myelodysplastic syndrome (MDS) and an IGH-CCNG1 in an AML. Our studies provide the first evidence that a once believed B-cell tumor-specific oncogenic mechanism is also present in myeloid tumors.

Case \#1: A 60-year-old female with a history of breast cancer presented with fatigue, bilateral flank pain, and hematuria. Complete blood count showed $24.82 \times 10^{9} / \mathrm{L}$ of white blood cells, $11.2 \mathrm{~g} / \mathrm{dL}$ of hemoglobin, $33.9 \%$ of hematocrit, and $68 \times 10^{\circ} / \mathrm{L}$ of platelets. The bone marrow

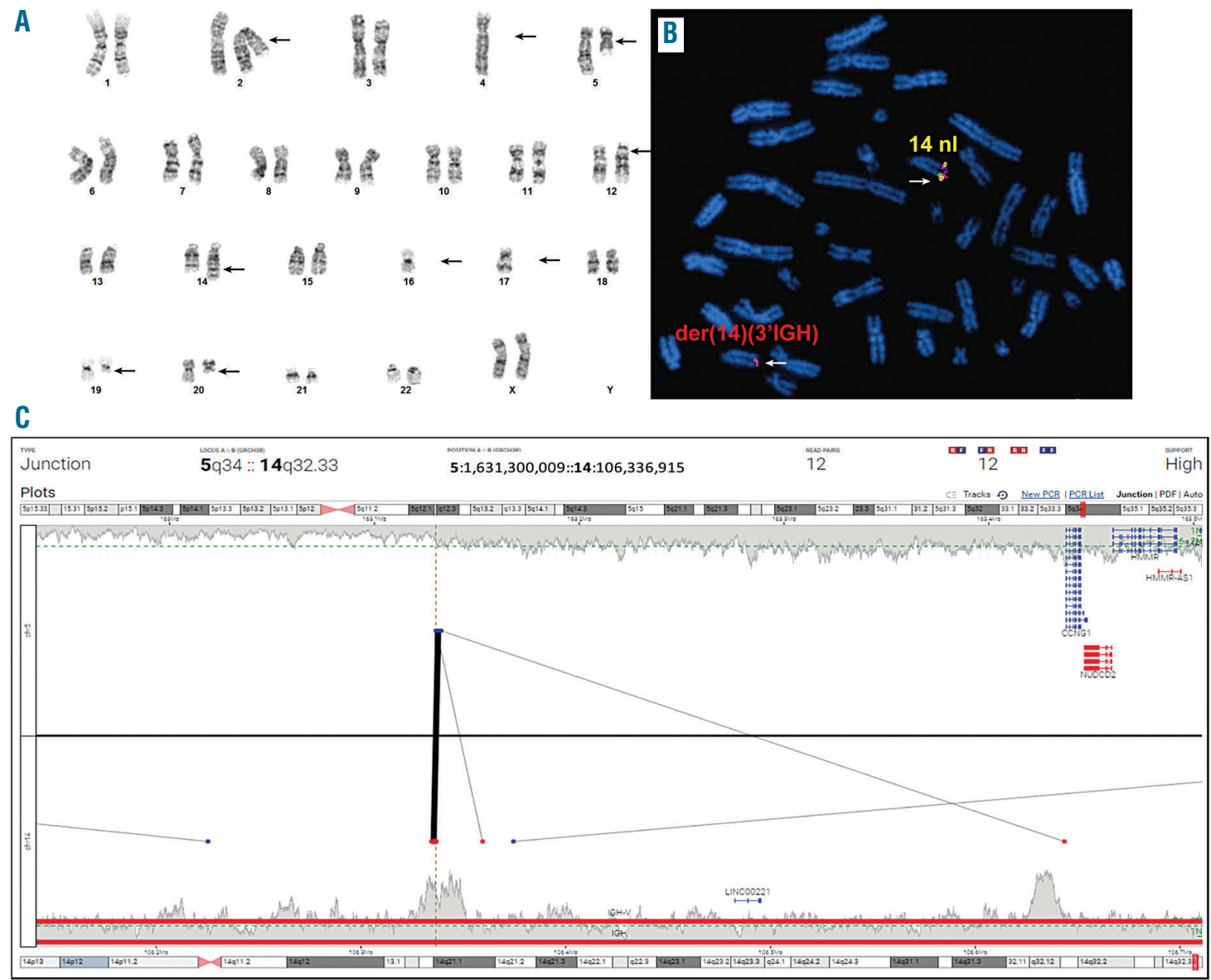

D

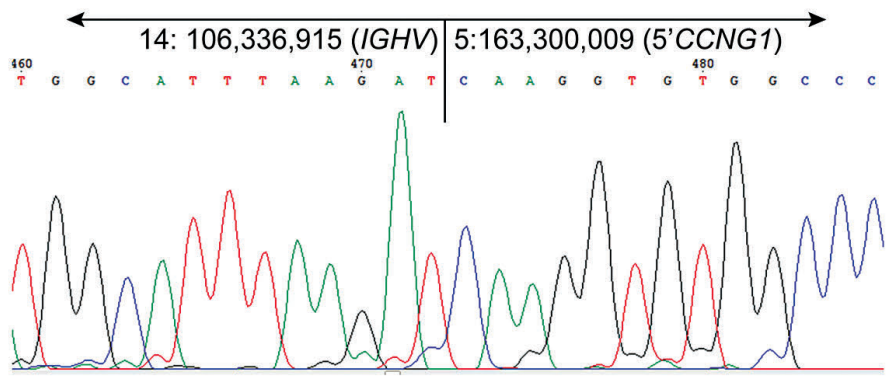

$\mathrm{E}$

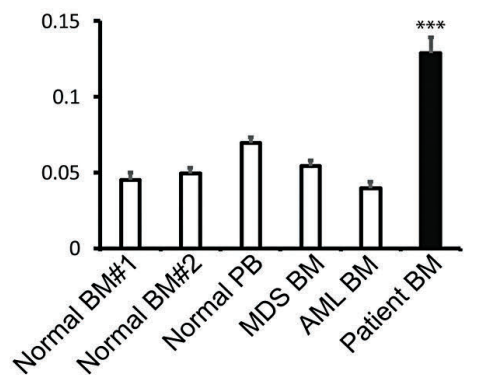

Figure 1. Acute myeloid leukemia with an IGH-CCNG1 rearrangement. (A) Chromosome analysis of the bone marrow aspirate showed a complex karyotype, including an unbalanced translocation involving chromosomes 5, 14 and 19. Arrows indicate clonal aberrations. (B) Fluorescence in situ hybridization (FISH) on abnormal metaphase showed the $3^{\prime} / G H$ (red) remaining on the derivative chromosome 14 and the 5 '/GH lost, consistent with an unbalanced IGH rearrangement. (C) A mate-pair next-generation sequencing (NGS) found an IGH-CCNG1 rearrangement (3'IGH-V3::5'CCNG1). (D) IGH-CCNG1 breakpoints were confirmed by PCR followed by Sanger sequencing. (E) The CCNG1 expression level was evaluated by a real-time quantitative RT-PCR with the patient's bone marrow, a normal blood sample, two normal bone marrow samples, a myelodysplastic syndrome (MDS) with a normal karyotype and an acute myeloid leukemia (AML) with a complex karyotype without $14 \mathrm{q}(\mathrm{IGH})$ rearrangement. CCNG1 expression was normalized to ACTB. ***: $P<0.001$. 


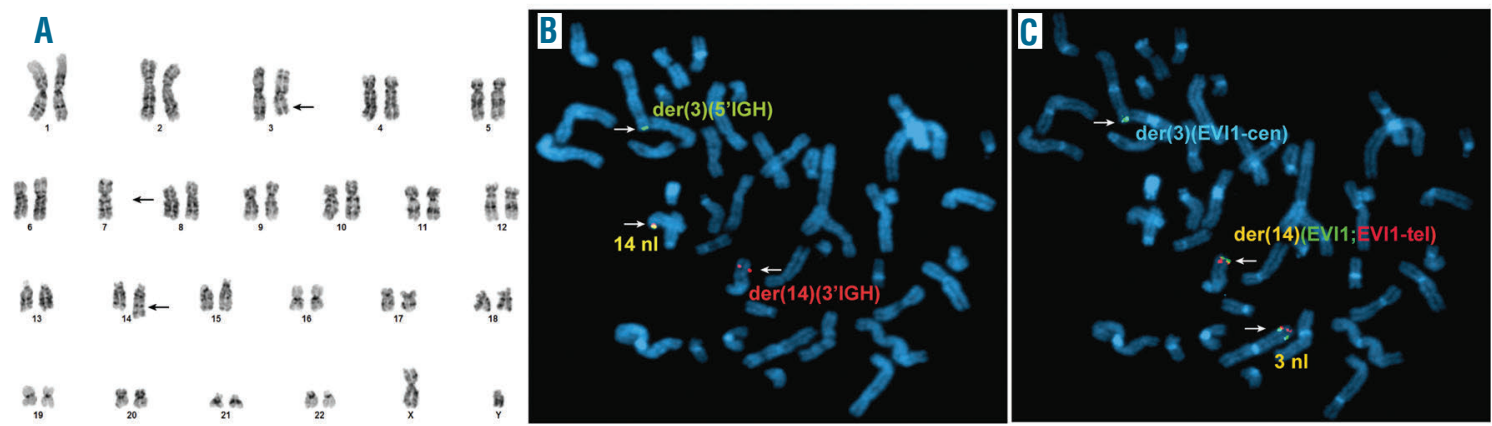

Figure 2. Myelodysplastic syndrome with an IGH-MECOM rearrangement. (A) Chromosome analysis of the bone marrow aspirate showed monosomy 7 and a balanced translocation $\mathrm{t}(3 ; 14)$. Arrows indicate clonal aberrations. (B) Fluorescence in situ hybridization (FISH) on an abnormal metaphase with a MECOM (EVI1) probe showed the centromeric MECOM (aqua) remaining on chromosome 3 and both the MECOM (green) and the telomeric MECOM (red) translocated to chromosome 14. (C) FISH on the same abnormal metaphase (after probe stripping) with a IGH probe showed the 3'IGH (red) remaining on chromosome 14 and the 5'/GH translocated to chromosome 3.

biopsy was markedly hypercellular with less than $5 \%$ fat. Approximately $80-90 \%$ of the cellularity was composed of intermediate-sized blasts. Flow cytometric analysis of a peripheral blood specimen demonstrated $23 \%$ of immature cells that was positive for CD45(dim), CD34, HLADR, the myeloid markers CD13(subset), CD56 (subset), CD33, CD15 (subset), CD11b, CD117 (subset), and CD123, but was negative for other monocytic, B- and T-lymphoid markers, consistent with myeloblasts. A targeted next-generation sequencing (NGS) gene panel for leukemia showed DNMT3A c.1322delG p.V441fs* (VAF, 55.5\%), TET2 c.5618T>C p.I1873T (85.4\%), and TP53 c.814G>A p.V272M (85.1\%), all common mutations in AML. Bone marrow cytogenetic analysis showed a complex karyotype with an unbalanced three way translocation involving chromosomes 5, 14 and 19, among other changes $(43, \mathrm{XX}, \operatorname{add}(2)(\mathrm{p} 23),-$ 4, der(5)t(5;14;19)(q34;q32;q13.2)del(5)(q14q34),i(11)(q1 $0), \operatorname{der}(12) \operatorname{del}(12)(\mathrm{p} 11.2)$ ? $\operatorname{dup}(12)(\mathrm{q} 14 \mathrm{q} 23), \operatorname{der}(14) \mathrm{t}(5 ; 14$; 19),-16,-17, $\operatorname{der}(19) \mathrm{t}(5 ; 14 ; 19), \operatorname{del}(20)(\mathrm{q} 11.2)$ [cp20] (Figure 1A). Because the translocation $\mathrm{t}(5 ; 14 ; 19)$ involved $14 \mathrm{q} 32$, the IGH locus, fluorescence in situ hybridization (FISH) was performed with an IGH split-apart probe. While the 3'IGH remained on the derivative chromosome 14 , the 5'IGH was lost, consistent with an unbalanced IGH rearrangement (Figure 1B). A mate-pair NGS ${ }^{1}$ showed that the chromosome 14 breakpoint was within IGH at the IGHV3-30, which fused to a chromosome 5 fragment approximately $308 \mathrm{~Kb}$ upstream of the 5' CCNG1 gene (NM_004060). The fusion led to a juxtaposition of the $3^{\prime} I G \bar{H}$, including the intact intragenic enhancer $(\mathrm{E} \mu)$ and the 3'-regulatory region (3'-RR), to CCNG1 on the der(14) (Figure 1C). The IGH-CCNG1 rearrangement was confirmed by PCR amplification and Sanger sequencing, using primers specific to IGH and CCNG1 (Figure $1 \mathrm{D}$ and Online Supplementary Table S1). A real-time quantitative RT-PCR with CCNG1-specific primers showed CCNG1 overexpression in the patient's bone marrow compared to normal blood and bone marrow specimens and myeloid tumors without 14q $(I G H)$ rearrangement (Figure 1E and Online Supplementary Table S2). The patient was diagnosed with therapy-related AML. She received a standard 7+3 regimen with no clinical response followed by another 10 day course of Decitabine, which also failed. The patient succumbed to the disease two months later.

Case \#2: A 46-year-old male with a history of acute promyelocytic leukemia presented with thrombocytopenia and anemia. The bone marrow biopsy showed a fragmented, moderately hypocellular marrow with $80 \%$ fat.
Erythroid elements were moderately proportionally decreased and exhibited maturation. Myeloid elements were moderately proportionally increased, exhibiting leftshifted maturation and including increased numbers of early and eosinophilic forms. Blasts comprised up to $10 \%$ of the marrow cellularity, as confirmed by immunoperoxidase study for CD34. A differential count of the bone marrow aspirate found $10 \%$ of blasts and dysplastic myeloid elements. Flow cytometry of the bone marrow showed that the immature cells were positive for CD45(dim), CD34, HLA-DR, myeloid markers CD13, CD33, CD117, and $11 \mathrm{~B}$ (subset), but that the bone marrow was negative for other monocytic, B- and T-lymphoid markers, consistent with myeloblasts. Bone marrow cytogenetics showed monosomy 7 and a balanced translocation between chromosomes 3 and 14 (45,XY,$7, t(3 ; 14)(q 26.2 ; q 32)[19] / 46, X Y[1])$ (Figure 2A). FISH assays on abnormal metaphases with a MECOM triplecolored probe confirmed a $M E C O M$ rearrangement, with part of the probe, including the intact $M E C O M$, translocated to chromosome 14 (Figure 2B). The cells were then hybridized to an IGH split-apart probe, which showed an $I G H$ rearrangement, with 3'IGH remaining on the chromosome 14 and the 5'IGH translocated to chromosome 3 (Figure 2C). Together these FISH results are consistent with an IGH-MECOM fusion. Unfortunately, no materials were available for mate-pair NGS or RT-PCR for further characterization of the rearrangement. The patient was diagnosed with therapy-related MDS, best regarded as refractory anemia with excess blasts-2. After four cycles of decitabine, his blast count was $<5 \%$ and he underwent a stem cell transplant (SCT) with his brother as the donor. He is now six years after SCT and healthy.

We presented here two cases of therapy-related myeloid tumors with $I G H$ rearrangements, which have never been reported before. $I G H$ rearrangement is one of the most common findings in mature B-cell tumors, which leads to a juxtaposition of the potent IGH enhancers nearby otherwise silent oncogenes, leading to their expression and activation. More than 40 genes are fused with $I G H$, with the common ones being $M Y C, B C L 2$ and BCL6, CCND1, FGFR3, as seen in Burkitt lymphoma, follicular lymphoma, mantle cell lymphoma, and multiple myeloma, respectively. These rearrangements are clinically important for the diagnosis and classification of these B-cell tumors. In B lymphocytes, $I G H$ enhancers interact with $I G H$ promoters to regulate a tissue-specific and temporally regulated expression. ${ }^{2}$ While the intragenic E $\mu$ enhancer controls the $\mathrm{V}(\mathrm{D}) \mathrm{J}$ recombination, the $3^{\prime} \mathrm{RR}$ controls class switch 
recombination (CSR) and somatic hypermutation (SHM). Early studies showed an unexpected V(D)J rearrangement in AML (not to be confused with the IGH rearrangement leading to gene fusion seen in our cases), ${ }^{3}$ suggesting that these non-B-cell tumors somehow activate the complex $I G H$ recombination and maturation process. IGH expression has subsequently been detected in several types of non-B-cell tumors, including breast cancer, colon cancer, lung cancer, hepatoma, cervical cancer and AML. ${ }^{4}$ In AML, the $\mathrm{V}(\mathrm{D}) \mathrm{J}$ recombination and the SHM of $\mathrm{V}$ regions were found in myeloblasts, but not in monocytes or neutrophils from healthy controls or patients with non-hematopoietic neoplasms. ${ }^{5}$ How these non-B-tumor cells activate an otherwise cell-specific IGH program is unknown. Several attributes of tumors may contribute to ectopic IGH expression. (1) Global changes in the epigenetic landscape are a hallmark of cancer, which may lead to local chromatin accessibility of the IGH locus. (2) Deregulation of the IGH enhancer-binding transcription factors is frequent in tumors, which may lead to ectopic activation of $I G H$ enhancers. Ets proteins and Ikros, both major regulators of B-cell development, are deregulated in myeloid tumors. ${ }^{6}$ OCT-2 is a co-activator of IGH enhancer $\mathrm{E} \mu$ and is expressed in AML with the $t(8 ; 21) ;^{7}$ and (3) The EpsteinBarr virus (EBV) infection can be seen in various tumors including AML. ${ }^{8}$ The EBV protein LMP1 increased the enhancer activity of IG $\kappa$ light chain by activating the NF$\kappa \mathrm{B}$ and AP-1 pathways in nasopharyngeal carcinoma cells. ${ }^{9}$ Similar activation mechanisms may also be present for the IGH locus.

$I G H$ is fused with two well-known oncogenes including $M E C O M$ and CCNG1 in myeloid tumors. MECOM is often rearranged in MDS and AML. The classic $\operatorname{inv}(3 q) / t(3 ; 3)$ bring the GATA2 distal hematopoietic enhancer (G2DHE) to the vicinity of MECOM, leading to its overexpression. ${ }^{10} \mathrm{MECOM}$ is a transcription factor that, when overexpressed, induces upregulation of cell cycle and blocks granulocytic differentiation of hematopoietic stem cells. ${ }^{11}$ CCNG1 is a non-canonical cyclin involved in the regulation of both Tp53 and cMYC. CCNG1 binds to Ser/Thr protein phosphatase 2A (PP2A), which dephosphorylates and activates mouse double minute 2 (Mdm2). Activated Mdm2 serves as an E3 ubiquitin ligase of Tp53, leading to its proteasome degradation (cyclin G1/Mdm2/Tp53 axis). ${ }^{12}$ CCNG1 also binds to CDK2 or CDK5, which phosphorylates and activates cMYC (cyclin G1/CDk2/c-Myc axis). ${ }^{13}$ Because our case with IGHCCNG1 rearrangement had monosomy 17 and a TP53 mutation, it is likely that the cyclin G1/CDk2/c-Myc axis, instead of cyclin G1/Mdm2/Tp53 axis, plays a role in its tumorigenesis. CCNG1 overexpression or amplification is found in many tumors. ${ }^{14}$ Clinical trials with a dominantnegative CCNG1 retroviral expression (DeltaRex-G) showed impressive results, including several metastatic tumors being cancer-free ten years after DeltaRex-G monotherapy. ${ }^{15}$ TP53 loss or mutation is relatively rare in myeloid tumors, suggesting an intact cyclin G1/Mdm2/p53 axis. MYC is located on chromosome 8. Trisomy 8 is one of the most common findings in myeloid tumors, which leads to MYC copy number gain. In addition, double minutes or homogeneously staining regions containing MYC amplification are often seen in AML. These studies are consistent with an active MYC signaling. It will be interesting to evaluate how frequent the CCNG1 is overexpressed in myeloid tumors, and whether an antiCCNG1 strategy such as DeltaRex-G is effective in treating myeloid tumors, a group of diseases typically with very poor prognosis.

In summary, we find that the $I G H$ rearrangement can happen in myeloid tumors. Similar to those in B-cell tumors, IGH rearrangements lead to the juxtaposition of the IGH enhancers to the vicinity of oncogenes, leading to their overexpression.

Amy W. Xiao, ${ }^{1}$ Yuyan Jia, ${ }^{1}$ Linda B. Baughn, ${ }^{2}$

Kathryn E. Pearce, ${ }^{2}$ Beth A. Pitel, Jon C. Aster,

Paola Dal Cin ${ }^{1}$ and Sheng Xiao

'Department of Pathology, Brigham and Women's Hospital, Harvard Medical School, Boston, MA and 'Division of Laboratory Genetics, Department of Laboratory Medicine and Pathology, Mayo Clinic Rochester, MN, USA

Correspondence: SHENG XIAO - sxiao@partners.org doi:10.3324/haematol.2020.246744

Information on authorship, contributions, and financial \& other disclosures was provided by the authors and is available with the online version of this article at www. haematologica.org.

\section{References}

1. Vasmatzis G, Johnson SH, Knudson RA, et al. Genome-wide analysis reveals recurrent structural abnormalities of TP63 and other p53related genes in peripheral T-cell lymphomas. Blood. 2012; 120(11):2280-2289

2. Roy AL, Sen R, Roeder RG. Enhancer-promoter communication and transcriptional regulation of Igh. Trends Immunol. 2011;32(11):532539.

3. Rovigatti U, Mirro J, Kitchingman G, Dahl G. Heavy chain immunoglobulin gene rearrangement in acute nonlymphocytic leukemia. Blood. 1984;63(5):1023-1027.

4. Oiu Y, Korteweg C, Chen Z, et al. Immunoglobulin G expression and its colocalization with complement proteins in papillary thyroid cancer. Mod Pathol. 2012;25(1):36-45.

5. Qiu X, Sun X, He Z, et al. Immunoglobulin gamma heavy chain gene with somatic hypermutation is frequently expressed in acute myeloid leukemia. Leukemia. 2013;27(1):92-99.

6. Marcucci G, Maharry K, Whitman SP, et al. High expression levels of the ETS-related gene, ERG, predict adverse outcome and improve molecular risk-based classification of cytogenetically normal acute myeloid leukemia: A cancer and leukemia group B study. J Clin Oncol. 2007;25(22):3337-3343.

7. Valbuena JR, Medeiros LJ, Rassidakis GZ, et al. Expression of B cellspecific activator protein/PAX5 in acute myeloid leukemia with $\mathrm{t}(8 ; 21)(\mathrm{q} 22 ; \mathrm{q} 22)$. Am J Clin Pathol. 2006;126(2):235-240.

8. Guan H, Miao H, Ma N, et al. Correlations between Epstein-Barr virus and acute leukemia. Med Virol. 2017;89(8):1453-1460.

9. Liu $\mathrm{H}$, Zheng H, Duan Z, et al. LMP1-augmented kappa intron enhancer activity contributes to upregulation expression of Ig kappa light chain via NF-kappaB and AP-1 pathways in nasopharyngeal carcinoma cells. Mol Cancer. 2009;8:92.

10. Yamazaki H, Suzuki M, Otsuki A, et al. A remote GATA2 hematopoietic enhancer drives leukemogenesis in inv(3)(q21;q26) by activating EVI1 expression. Cancer Cell. 2014;25(4):415-427.

11. Morishita K, Parganas E, Matsugi T, et al. Expression of the Evi-1 zinc finger gene in 32Dc13 myeloid cells blocks granulocytic differentiation in response to granulocyte colony-stimulating factor. Mol Cell Biol. 1992;12(1):183-189.

12. Okamoto K, Li H, Jensen MR, et al. Cyclin G recruits PP2A to dephosphorylate Mdm2. Mol Cell. 2002;9(4):761-771.

13. Seo HR, Kim J, Bae S, Soh JW, Lee YS. Cdk5-mediated phosphorylation of c-Myc on Ser-62 is essential in transcriptional activation of cyclin B1 by cyclin G1. J Biol Chem. 2008;283(23):15601-15610.

14. Ravicz J, Gordon EM, Szeto C, et al. Differential expression of human cyclin G1 (CCNG1) in cancer: A novel biomarker in development for CCNG1 inhibitor therapy. J Clin Oncol. 2018;e24315.

15. Kim S, Federman N, Gordon EM, Hall FL, Chawla SP. Rexin-G®, a tumor-targeted retrovector for malignant peripheral nerve sheath tumor: A case report. Mol Clin Oncol. 2017;6(6):861-865. 